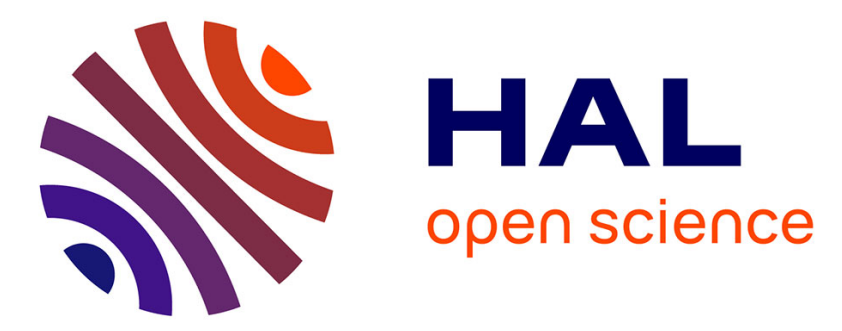

\title{
Consensus-based formation control of nonholonomic robots without velocity measurements
}

\author{
Tonatiuh Hernández, Antonio Loria, Emmanuel Nuño, Elena Panteley
}

\section{To cite this version:}

Tonatiuh Hernández, Antonio Loria, Emmanuel Nuño, Elena Panteley. Consensus-based formation control of nonholonomic robots without velocity measurements. ECC 2020 - European Control Conference, May 2020, St. Petersbourg, Russia. 10.23919/ecc51009.2020.9143841 . hal-03101538

\section{HAL Id: hal-03101538 \\ https://hal.science/hal-03101538}

Submitted on 7 Jan 2021

HAL is a multi-disciplinary open access archive for the deposit and dissemination of scientific research documents, whether they are published or not. The documents may come from teaching and research institutions in France or abroad, or from public or private research centers.
L'archive ouverte pluridisciplinaire HAL, est destinée au dépôt et à la diffusion de documents scientifiques de niveau recherche, publiés ou non, émanant des établissements d'enseignement et de recherche français ou étrangers, des laboratoires publics ou privés. 


\title{
Consensus-based formation control of nonholonomic robots without velocity measurements
}

\author{
Tonatiuh Hernández Antonio Loría Emmanuel Nuño Elena Panteley
}

\begin{abstract}
We present a novel smooth time-varying controller for the formation control of nonholonomic robots. The controller is of proportional-plus-derivative type, but it does not require velocity measurements; the latter are replaced by approximate differentiation. To overcome the difficulty imposed by the nonholonomy, the controller relies on a $\delta$-persistentlyexciting term which, roughly speaking, excites all the systems' modes while the position errors are away from zero. Assuming that the interconnection static graph is undirected and connected, it is showed that the nonholonomic robots converge to a given formation pattern around a rendez-vous point and their orientations tend to a common angle. To the best of our knowledge, this is the first smooth controller for formation consensus control of nonholonomic systems, via a output feedback.
\end{abstract}

Index Terms-Formation Control, Nonholonomic Robots, Output Feedback

\section{INTRODUCTION}

The basic synchronization behavior in networks of dynamical agents is consensus, in which case the states of all the agents converge to a common agreement value [1], [2]. From the application point of view, consensus should be achieved by only sharing (part of) the agent's state with their corresponding neighbors using a given interconnection topology [3].

The complexity of consensus control problems stems, primarily, from network properties as the nature of the interconnections, the topology, etc. However, to no lesser extent, such complexity also resides in the agent dynamics that compose the network [4], [5], [6]. An example of a strongly nonlinear agent is a nonholonomic mobile robot. Indeed, the nonholonomic restriction prevents the applicability of smooth autonomous controllers to solve consensus problems [7]. However, due to the different applications of consensus of mobile robots, it is physically appealing to design a decentralized controller for a swarm of mobile robots to converge to a predetermined formation pattern whose location in the plane is determined by an a priori unknown center and with a common non pre-determined orientation. In doing so, they

Tonatiuh Hernández and Emmanuel Nuño are with the Department of Computer Science at the University of Guadalajara. Guadalajara, Mexico (e-mail: tonatiuh.hernandez@academicos.udg.mx). Antonio Loría and Elena Panteley are with the CNRS, at Laboratoire des signaux et systèmes, L2S-CentraleSupélec, Gif-sur-Yvette, France; (e-mail: \{antonio.loria\} \{elena.panteley\}@ centralesupelec.fr).

This work has been partially supported by the Mexican CONACyT Basic Scientific Research grant CB-282807, This article is supported by Government of Russian Federation (grant 074-U01), by the French National Research Agency (ANR) via the project "HANDY" - contract number: ANR-18-CE40-0010, and by CEFIPRA under the grant number 6001-A. achieve a formation. The goal involves stabilizing each of the nonholonomic robot, in position and orientation, to a point.

Different decentralized solutions for consensus problems in networks of nonholonomic robots are proposed in the literature, e.g., [8], [9], [10], [11]. Most of the works proposing smooth time-varying controllers for nonholonomic vehicles, however, employ a simplified first-order model that considers exclusively the kinematics and, with the exception of [12], all of those that also consider the dynamics require velocity measurements; see for example [13], [14]. For leader-follower tracking formation control in the recent work [12] a velocity super-twisting observer, which ensures that the estimated velocities converge in finite-time to the real values, together with a discontinuous sliding-mode controller to achieve a formation control objective is used. However, super-twisting-based differentiators rely on the conservative assumption that the acceleration is bounded and discontinuous controllers may present undesirable chattering.

In this paper we propose a novel smooth time-varying controller that globally asymptotically stabilizes the network of nonholonomic robots at a given formation pattern and with a common orientation. To the best of our knowledge, this is the first article where a smooth controller for consensus of nonholonomic robots, via an output-feedback controller is proposed. The formation-consensus goal is achieved for swarms of robots communicating through reliable channels and interconnected in an undirected, connected graph.

The proposed dynamic controller is fairly simple. It is of the proportional-plus-derivative type, except that velocity measurements are replaced by an approximate-differentiation filter. Due to its passivity properties, such controller has been proved to stabilize the system, globally, in set-point control tasks [15], in tracking control [16], and even under the presence of measurement delays [17]. Now, while for Euler-Lagrange systems with holonomic constraints, a simple autonomous proportional-derivative-plusapproximate-differentiation controller suffices, to achieve stabilization for nonholonomic robots the controller must also rely on the injection of appropriate excitation. This is provided in the form of so-called $\delta$-persistency of excitation, a property that is necessary and sufficient for the uniform asymptotic stability of nonlinear time-varying systems [18]. In the control of nonholonomic systems $\delta$-persistently excitation has been used, starting with [19], in several succeeding articles, see e.g., [20].

The rest of this paper is organized as follows. In next section we present the system's model and formulate the 
formation-consensus problem. In Section III we present our main result. In Section IV we illustrate our theoretical findings via a numerical example and in Section $\mathrm{V}$ we conclude with some remarks.

\section{MODEL AND PROBLEM FORMULATION}

Notation. $\mathbb{R}:=(-\infty, \infty), \mathbb{R}_{\geq 0}:=[0, \infty)$ and $\mathbb{R}_{>0}:=$ $(0, \infty) .|\mathbf{x}|$ stands for the standard Euclidean norm of vector x. $\mathbf{1}_{k}$ and $\mathbf{0}_{k}$ represent column vectors of size $k$ with all entries equal to one and to zero, respectively. $\otimes$ represents the standard Kronecker product. For any function $\mathbf{f}: \mathbb{R}_{\geq 0} \rightarrow$ $\mathbb{R}^{n}$, the $\mathcal{L}_{\infty}$-norm is defined as $\|\mathbf{f}\|_{\infty}:=\sup _{t>0}|\mathbf{f}(t)|, \mathcal{L}_{2}$-norm as $\|\mathbf{f}\|_{2}:=\left(\int_{0}^{\infty}|\mathbf{f}(t)|^{2} d t\right)^{1 / 2}$. The $\mathcal{L}_{\infty}$ and $\mathcal{L}_{2}$ spaces are defined as $\left\{\mathbf{f}: \mathbb{R}_{\geq 0} \rightarrow \mathbb{R}^{n} \mid\|\mathbf{f}\|_{\infty}<\infty\right\}$ and $\left\{\mathbf{f}: \mathbb{R}_{\geq 0} \rightarrow\right.$ $\left.\mathbb{R}^{n} \mid\|\mathbf{f}\|_{2}<\infty\right\}$, respectively.

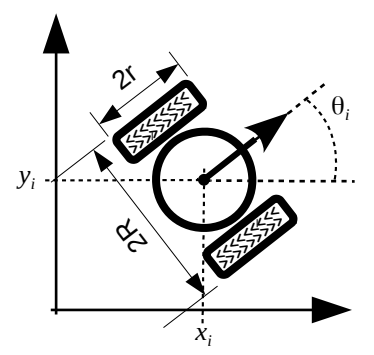

Fig. 1. Schematic representation of a differential wheeled mobile robot

We consider a swarm of $N$ nonholonomic vehicles, of the kind illustrated in Figure 1, which are modeled as differential-drive robots that move in the Cartesian $x y$-plane with three degrees of freedom, two translations and one rotation. For each $i \leq N$, the Cartesian coordinates determining the position of the $i$ th robot on the plane are denoted $\mathbf{z}_{i}:=\left[\begin{array}{ll}x_{i} & y_{i}\end{array}\right]^{\top} \in \mathbb{R}^{2}$ and the orientation with respect to the horizontal axis is denoted by $\theta_{i} \in \mathbb{R}$. Furthermore, we define $\boldsymbol{\delta}_{i} \in \mathbb{R}^{2}, \boldsymbol{\delta}_{i}=\left[\begin{array}{ll}\delta_{i x} & \delta_{i y}\end{array}\right]^{\top}$ as the relative desired translation of the $i$ th-robot with regards to the a priori undetermined centre $\mathbf{z}_{c}:=\left[\begin{array}{ll}x_{c} & y_{c}\end{array}\right]^{\top}$ of a given formation pattern. Thus, the corresponding position of the $i$ th-robot translated to the centre of the desired formation is $\overline{\mathbf{z}}_{i}:=\mathbf{z}_{i}-\boldsymbol{\delta}_{i}$. See Figure 2 for an illustration.

The kinematic model of these nonholonomic robots, under the common non-slippage assumption, is given by

$$
\begin{aligned}
\dot{\mathbf{z}}_{i} & =\varphi\left(\theta_{i}\right) v_{i}, \\
\dot{\theta}_{i} & =\omega_{i},
\end{aligned}
$$

where $\varphi\left(\theta_{i}\right):=\left[\cos \left(\theta_{i}\right) \sin \left(\theta_{i}\right)\right]^{\top}$ and $v_{i}, \omega_{i} \in \mathbb{R}$ are the linear and angular velocities of the center of mass of the $i$ th-robot, respectively.

We assume that for each robot, the geometrical center and the center of mass are located at the same point $\mathbf{z}_{\mathbf{i}}:=$ $\left[x_{i}, y_{i}\right]^{\top}$ and thus its corresponding dynamic behavior is governed by

$$
\left[\begin{array}{cc}
m_{i} & 0 \\
0 & I_{i}
\end{array}\right]\left[\begin{array}{c}
\dot{v}_{i} \\
\dot{\omega}_{i}
\end{array}\right]=\frac{1}{r_{i}}\left[\begin{array}{cc}
1 & 1 \\
2 R_{i} & -2 R_{i}
\end{array}\right] \boldsymbol{\tau}_{i}
$$

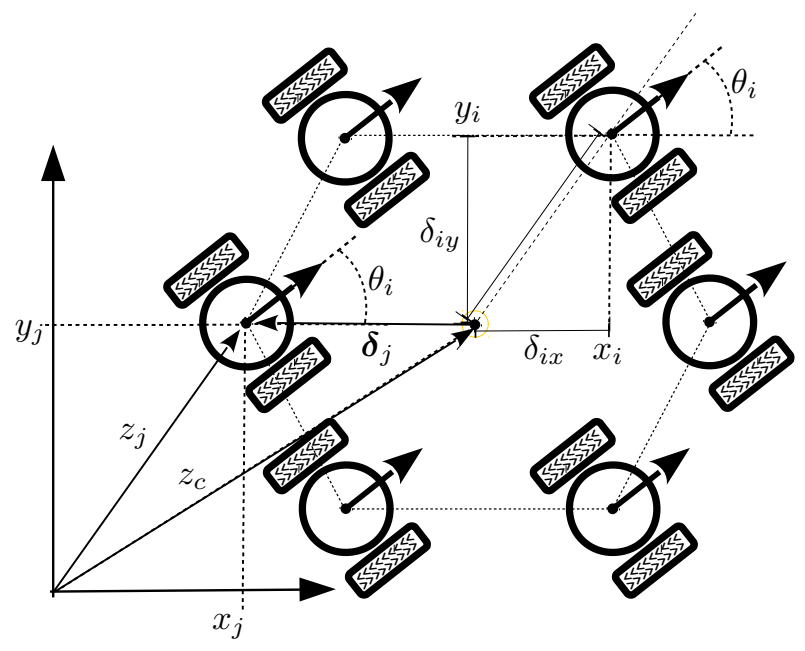

Fig. 2. Example of formation pattern with common orientation

where $m_{i}$ is the mass; $I_{i}$ is the moment of inertia; $R_{i}$ is the distance between point $\mathbf{z}_{\mathbf{i}}$ and the wheels; $r_{i}$ the radius of the wheels; and $\tau_{i}$ is the control input torque of the left and right wheels, i.e., $\boldsymbol{\tau}_{i}=\left[\tau_{l i}, \tau_{r i}\right]^{\top}-$ see Fig. 1 .

The interconnection of the agents is modeled as a undirected weighted graph via the Laplacian matrix $\mathbf{L}:=\left[l_{i j}\right] \in$ $\mathbb{R}^{N \times N}$, whose elements are defined as

$$
\ell_{i j}=\left\{\begin{array}{cc}
\sum_{k \in \mathcal{N}_{i}} a_{i k} & i=k \\
-a_{i k} & i \neq k
\end{array}\right.
$$

where $\mathcal{N}_{i}$ is the set of agents transmitting information to the $i$-th robot. Each interconnection weight $a_{i j}$ is associated to the edge $(i, j)$. Since the interconnection graph is undirected $a_{i j}=a_{j i}$ and hence the Laplacian matrix is symmetric. If the pair of nodes $(i, j)$ is connected then $a_{i j}>0$ otherwise $a_{i j}=0$. The Laplacian matrix has zero row sums and, consequently, zero is an eigenvalue associated with the eigenvector $\mathbf{1}_{N}:=\left[\begin{array}{lll}1 & \cdots & 1\end{array}\right]^{\top}$. Furthermore, if in addition the graph is connected, $\mathbf{L}$ is symmetric with only one zero eigenvalue and the rest of its spectrum is strictly positive. Thus, $\mathbf{L}$ is symmetric positive semidefinite.

\section{CONSEnsus-BASEd Formation CONTROL}

Consider a swarm of $N$ differential wheeled robots modeled as (1) and (2) under that each robot possesses position and orientation sensors to obtain $z_{i}$ and $\theta_{i}$, but the respective forward and angular velocities $v_{i}$ and $\omega_{i}$ are not available from direct measurements. Under these condition, the goal is to design a decentralized controller such that, given a desired formation pattern, all robots asymptotically agree on their relative Cartesian positions and orientation, independently of the initial conditions, i.e., there exists $\mathbf{z}_{c} \in \mathbb{R}^{2}$ and $\theta_{c} \in \mathbb{R}$ 
such that

$$
\lim _{t \rightarrow \infty}\left[\begin{array}{c}
\overline{\mathbf{z}}_{i}(t) \\
\theta_{i}(t) \\
v_{i}(t) \\
\omega_{i}(t)
\end{array}\right]=\left[\begin{array}{c}
\mathbf{z}_{c} \\
\theta_{c} \\
0 \\
0
\end{array}\right], \quad \forall i \leq N
$$

\section{- see Figure 2.}

This problem may be solved using a decentralized controller involving a consensus correction term, which is defined in function of the Cartesian position error and orientation error of each agent with respect to its neighbors. These are given by

$$
\begin{aligned}
\mathbf{e}_{i} & =\sum_{j \in \mathcal{N}_{i}} a_{i j}\left(\overline{\mathbf{z}}_{i}-\overline{\mathbf{z}}_{j}\right), \\
e_{\theta i} & =\sum_{j \in \mathcal{N}_{i}} a_{i j}\left(\theta_{i}-\theta_{j}\right) .
\end{aligned}
$$

Now, to compact the notation, we collect the positions and orientations in the vectors $\overline{\mathbf{z}}:=\left[\begin{array}{lll}\overline{\mathbf{z}}_{1}^{\top} \cdots & \overline{\mathbf{z}}_{N}^{\top}\end{array}\right]^{\top}$ and $\boldsymbol{\theta}:=$ $\left[\theta_{1} \cdots \theta_{N}\right]^{\top}$. Similarly, the position and orientation errors are collected in the vectors $\mathbf{e}=\left[\begin{array}{lll}\mathbf{e}_{1}^{\top} & \cdots & \mathbf{e}_{N}^{\top}\end{array}\right]^{\top}$ and $\mathbf{e}_{\theta}=$ $\left[\begin{array}{lll}e_{\theta 1} & \cdots & e_{\theta N}\end{array}\right]^{\top}=\mathbf{L} \boldsymbol{\theta}$, respectively. For further development, we also stress that

$$
e=\left(\mathbf{L} \otimes I_{2}\right) \overline{\mathbf{z}}, \quad I_{2}:=\left[\begin{array}{ll}
1 & 0 \\
0 & 1
\end{array}\right]
$$

Since $\mathbf{1}_{N}$ is the associated eigenvector of the single zero eigenvalue of the Laplacian matrix, the control objective is achieved provided that

$$
\lim _{t \rightarrow \infty}\left[\begin{array}{c}
\boldsymbol{e}_{i}(t) \\
\boldsymbol{e}_{\theta i}(t) \\
v_{i}(t) \\
\omega_{i}(t)
\end{array}\right]=\left[\begin{array}{l}
0 \\
0 \\
0 \\
0
\end{array}\right], \quad \forall i \leq N
$$

\section{- cf. Eq. (4).}

The first step in the control design involves the following inner control-loop

$$
\left[\begin{array}{c}
\tau_{r i} \\
\tau_{l i}
\end{array}\right]=\frac{r_{i}}{2}\left[\begin{array}{cc}
m_{i} & I_{i} / 2 R_{i} \\
m_{i} & -I_{i} / 2 R_{i}
\end{array}\right]\left[\begin{array}{c}
u_{v i} \\
u_{\omega i}
\end{array}\right]
$$

which yields

$$
\begin{aligned}
\dot{v}_{i} & =u_{v i} \\
\dot{\omega}_{i} & =u_{\omega i} .
\end{aligned}
$$

Then, we introduce the new control inputs, $u_{v i}$ and $u_{\omega i}$, given by

$$
\begin{aligned}
& u_{v i}:=-d_{v i} \varphi^{\top}\left(\theta_{i}\right) \boldsymbol{\vartheta}_{v i}-p_{v i} \varphi^{\top}\left(\theta_{i}\right) \mathbf{e}_{i} \\
& u_{\omega i}:=-d_{\omega i} \vartheta_{\omega i}-p_{\omega i} e_{\theta i}+\alpha_{i}\left(t, \theta_{i}, \mathbf{e}_{i}\right),
\end{aligned}
$$

where $\varphi\left(\theta_{i}\right):=\left[\cos \left(\theta_{i}\right), \sin \left(\theta_{i}\right)\right]^{\top}, d_{v i}, p_{v i}, d_{\omega i}$, and $p_{\omega i}$ are positive scalar control gains, and the variables $\boldsymbol{\vartheta}_{v i}$ and $\boldsymbol{\vartheta}_{\omega i}$ are defined as the outputs of the dynamical systems

$$
\begin{aligned}
\dot{\mathbf{q}}_{v i} & =-a_{v i}\left(\mathbf{q}_{v i}+\overline{\mathbf{z}}_{i}\right), \quad a_{v i}>0 \\
\boldsymbol{\vartheta}_{v i} & =\mathbf{q}_{v i}+\overline{\mathbf{z}}_{i},
\end{aligned}
$$

and

$$
\begin{aligned}
\dot{q}_{\omega i} & =-a_{\omega i}\left(q_{\omega i}+\theta_{i}\right), \quad a_{\omega i}>0 \\
\vartheta_{\omega i} & =q_{\omega i}+\theta_{i},
\end{aligned}
$$

respectively.

The function $\alpha_{i}$ is designed to be a $\delta$-persistently exciting function [18]. Let

$$
\alpha_{i}\left(t, \theta_{i}, \mathbf{e}_{i}\right):=k_{\alpha i} f_{i}(t) \varphi^{\perp \top}\left(\theta_{i}\right) \mathbf{e}_{i},
$$

where $k_{\alpha i}>0, \varphi^{\perp}\left(\theta_{i}\right)=\left[\begin{array}{ll}-\sin \left(\theta_{i}\right) & \cos \left(\theta_{i}\right)\end{array}\right]^{\top}$ is the annihilator of $\boldsymbol{\varphi}\left(\theta_{i}\right)$, i.e., $\boldsymbol{\varphi}^{\top}\left(\theta_{i}\right) \boldsymbol{\varphi}^{\perp}\left(\theta_{i}\right)=\boldsymbol{\varphi}^{\perp \top}\left(\theta_{i}\right) \boldsymbol{\varphi}\left(\theta_{i}\right)=$ 0 . The function $f_{i}: \mathbb{R}_{>0} \rightarrow \mathbb{R}$ is constructed to be twice continuously differentiable, and such that $f_{i}, \dot{f}_{i}, \ddot{f}_{i} \in \mathcal{L}_{\infty}$, $\lim _{t \rightarrow \infty} f_{i}(t) \neq 0$, and $\lim _{t \rightarrow \infty} \dot{f}_{i}(t) \neq 0$. This smooth timevarying function is included in the controller in order to overcome the obstacle that poses the robots' nonholonomy to asymptotic stabilization [21], [7].

The controller (7) is reminiscent of proportional-derivative based controllers that rely on velocity measurements [13]. In the current scenario, however, since only position and orientation are available for measurement, the controllers rely on approximate differentiation of the Cartesian positions $\overline{\mathbf{z}}_{i}$ and orientation $\theta_{i}$. These filter dynamics follow the seminal idea reported in [15].

Under the action of the controller (7) the closed-loop equations are given by the two interconnected systems,

$$
\Sigma_{v i}:\left\{\begin{aligned}
\dot{\mathbf{z}}_{i} & =\boldsymbol{\varphi}\left(\theta_{i}\right) v_{i}, \\
\dot{v}_{i} & =-d_{v i} \boldsymbol{\varphi}^{\top}\left(\theta_{i}\right) \boldsymbol{\vartheta}_{v i}-p_{v i} \boldsymbol{\varphi}^{\top}\left(\theta_{i}\right) \mathbf{e}_{i}, \\
\dot{\boldsymbol{\vartheta}}_{v i} & =-a_{v i} \boldsymbol{\vartheta}_{v i}+\boldsymbol{\varphi}\left(\theta_{i}\right) v_{i}
\end{aligned}\right.
$$

and

$$
\Sigma_{\omega i}:\left\{\begin{aligned}
\dot{\theta}_{i} & =\omega_{i} \\
\dot{\omega}_{i} & =-d_{\omega i} \vartheta_{\omega i}-p_{\omega i} e_{\theta i}+\alpha_{i}\left(t, \theta_{i}, \mathbf{e}_{i}\right), \\
\dot{\vartheta}_{\omega i} & =-a_{\omega i} \vartheta_{\omega i}+\omega_{i} .
\end{aligned}\right.
$$

On one hand, since $\varphi(\cdot)$ is bounded, system $\Sigma_{v i}$ may be considered as if it is decoupled from $\Sigma_{\omega i}$. Hence forming a cascaded system [22]. On the other hand, system $\Sigma_{\omega i}$ can be seen as a stable "filter" with a $\delta$-persistently-exciting input $\alpha_{i}$, i.e., $\alpha_{i}$ is persistently exciting for all $\mathbf{e}_{i} \neq 0$ [19]. Such stabilization mechanism overcomes the effects of the nonholonomy - $c f$. [19].

The excitation in $\alpha_{i}$ is propagated throughout the system $\Sigma_{v i}$ via $\theta_{i}$, thereby ensuring the convergence of $\mathbf{e}_{i}$ to zero and, consequently, of $\alpha_{i}$ itself. That is, the excitation is maintained while, and only while the errors $e_{i}$ are away from the origin.

We are now ready to state the main result.

Theorem 1: Consider a swarm of $N$ differential wheeled robots modeled as (1) and (2) and suppose that velocities $v_{i}$ and $\omega_{i}$ are not available for measurement. Then, if the interconnection graph is undirected and connected, the controller given by (6)-(9) ensures that (4) holds for all initial conditions. 
Proof: Consider the following function

$$
\mathcal{V}:=\frac{1}{2} \sum_{i=1}^{N}\left(\frac{1}{p_{v i}} v_{i}^{2}+\frac{d_{v i}}{p_{v i}}\left|\boldsymbol{\vartheta}_{v i}\right|^{2}+\frac{1}{2} \sum_{j \in \mathcal{N}_{i}} a_{i j}\left|\overline{\mathbf{z}}_{i}-\overline{\mathbf{z}}_{j}\right|^{2}\right)
$$

which is positive definite and radially unbounded in the space of $\left(v, \boldsymbol{\vartheta}_{v}, \boldsymbol{e}\right)$. Evaluating the total derivative, $\dot{\mathcal{V}}$, along the trajectories of $\Sigma_{v i}$ in (11), we obtain

$$
\dot{\mathcal{V}}=-\sum_{i=1}^{N} \frac{d_{v i} a_{v i}}{p_{v i}}\left|\boldsymbol{\vartheta}_{v i}\right|^{2}
$$

for which we used [4, Lemma 6.1] to obtain

$\frac{1}{2} \sum_{i=1}^{N} \sum_{j \in \mathcal{N}_{i}} a_{i j}\left(\dot{\overline{\mathbf{z}}}_{i}-\dot{\overline{\mathbf{z}}}_{j}\right)^{\top}\left(\overline{\mathbf{z}}_{i}-\overline{\mathbf{z}}_{j}\right)=\sum_{i=1}^{N} \sum_{j \in \mathcal{N}_{i}} a_{i j} \dot{\overline{\mathbf{z}}}_{i}^{\top}\left(\overline{\mathbf{z}}_{i}-\overline{\mathbf{z}}_{j}\right)$.

Since $\mathcal{V} \geq 0$ and $\dot{\mathcal{V}} \leq 0$ then $\boldsymbol{\vartheta}_{v i} \in \mathcal{L}_{2}$ and $\overline{\mathbf{z}}_{i}-$ $\overline{\mathbf{z}}_{j}, v_{i}, \boldsymbol{\vartheta}_{v i} \in \mathcal{L}_{\infty}$, for any $i \leq N$ and $j \in \mathcal{N}_{i}$. From (11), it also holds that $\dot{v}_{i}, \dot{\vartheta}_{v i} \in \mathcal{L}_{\infty}$.

Consider now the function

$$
\mathcal{W}:=\frac{1}{2} \sum_{i=1}^{N}\left(\frac{1}{p_{\omega i}} \omega_{i}^{2}+\frac{d_{\omega i}}{p_{\omega i}} \vartheta_{\omega i}^{2}+\frac{1}{2} \sum_{j \in \mathcal{N}_{i}} a_{i j}\left|\theta_{i}-\theta_{j}\right|^{2}\right)
$$

and evaluate its total derivative, $\dot{\mathcal{W}}$, along the trajectories of $\Sigma_{\omega}$ in (12) with $\alpha_{i}=0$. We obtain

$$
\dot{\mathcal{W}}=-\sum_{i=1}^{N} \frac{d_{\omega i} a_{\omega i}}{p_{\omega i}} \vartheta_{\omega i}^{2} \leq 0
$$

which implies that $\vartheta_{\omega i} \in \mathcal{L}_{2} \cap \mathcal{L}_{\infty}$, while $\omega_{i}$ and $\left|\theta_{i}-\theta_{j}\right| \in$ $\mathcal{L}_{\infty}$. From (12), with $\alpha_{i}=0$, we have that boundedness of these signals implies that $\dot{\omega}_{i}$ and $\dot{\vartheta}_{\omega i}$ are also bounded. Therefore, after Babălat's Lemma we have

$$
\lim _{t \rightarrow \infty} \vartheta_{\omega i}(t)=0
$$

Next, invoking Lemma 1 in the Appendix, on the system (16) as the last equation in (12), with state $\mathbf{x}=\vartheta_{\omega i}$ and input $\mathbf{u}=\omega_{i}$ we conclude that, also,

$$
\lim _{t \rightarrow \infty} \omega_{i}(t)=0
$$

Moreover,

$$
\lim _{t \rightarrow \infty} \int_{0}^{t} \dot{\omega}_{i}(t)=\lim _{t \rightarrow \infty} \omega_{i}(t)-\omega_{i}(0)=-\omega_{i}(0)
$$

and, since $\ddot{\omega}_{i}=-d_{\omega i} \dot{\vartheta}_{\omega i}-p_{\omega i} \sum_{j \in \mathcal{N}_{i}} a_{i j}\left(\omega_{i}-\omega_{j}\right), \ddot{\omega}_{i} \in \mathcal{L}_{\infty}$. Hence, $\dot{\omega}_{i}$ is uniformly continuous and invoking Barbălat Lemma we conclude that

$$
\lim _{t \rightarrow \infty} \dot{\omega}_{i}(t)=0
$$

so, from (12) with $\alpha_{i} \equiv 0$, we obtain $\lim _{t \rightarrow \infty} e_{\theta i}(t)=0$. In summary, provided that $\alpha_{i} \equiv 0$, we have $\left(\omega_{i}, \theta_{i}, \vartheta_{\theta i}\right) \rightarrow$ $\left(0, \theta_{c}, 0\right)$.

If $\alpha_{i} \not \equiv 0$, note that since $f_{i}(t), \mathbf{e}_{i}$ and $\varphi^{\perp}\left(\theta_{i}\right)$ are bounded, so is $\alpha_{i}$-see Eq. (10). This and the fact that
$\Sigma_{\omega}$ is a marginally stable linear time invariant system imply with input $\alpha_{i}$ that $\dot{\omega}_{i}, \omega_{i} \in \mathcal{L}_{\infty}$, by Proposition 3 in [23], so $e_{\theta i} \in \mathcal{L}_{\infty}$.

Next, we center our attention in the proof that $\mathbf{e}_{i}$ converges to zero. So far we have established that $v_{i}, \dot{v}_{i}, \boldsymbol{\vartheta}_{v i}, \dot{\boldsymbol{\vartheta}}_{v i}, \mathbf{e}_{i}, \omega_{i}, \dot{\omega}_{i}$ and $e_{\theta i}$ are bounded and that $\vartheta_{v i}, \vartheta_{\omega i} \in \mathcal{L}_{2}$.

Now, since $\frac{d}{d t} \boldsymbol{\varphi}\left(\theta_{i}\right)=\boldsymbol{\varphi}^{\perp}\left(\theta_{i}\right) \omega_{i}$, boundedness of $\dot{\boldsymbol{\vartheta}}_{v i}, \dot{v}_{i}$ and $\omega_{i}$ imply boundedness of $\ddot{\vartheta}_{v i}$. Therefore, invoking Lemma 1 in the Appendix, with $\mathbf{x}=\boldsymbol{\vartheta}_{v i}$, which satisfies $\boldsymbol{\vartheta}_{v i} \rightarrow 0$, and $\mathbf{u}=\boldsymbol{\varphi}\left(\theta_{i}\right) v_{i}$, we conclude that

$$
\lim _{t \rightarrow \infty} \varphi\left(\theta_{i}\right) v_{i}(t)=0
$$

so $\lim _{t \rightarrow \infty} v_{i}(t)=0$.

On the other hand,

$$
\lim _{t \rightarrow \infty} \int_{0}^{t} \dot{v}_{i}(t)=\lim _{t \rightarrow \infty} v_{i}(t)-v_{i}(0)=-v_{i}(0)
$$

and

$\ddot{v}_{i}=-\boldsymbol{\varphi}^{\top}\left(\theta_{i}\right)\left[d_{v i} \dot{\boldsymbol{\vartheta}}_{v i}+p_{v i} \dot{\mathbf{e}}_{i}\right]-\omega_{i} \boldsymbol{\varphi}^{\perp \top}\left(\theta_{i}\right)\left[d_{v i} \boldsymbol{\vartheta}_{v i}+p_{v i} \mathbf{e}_{i}\right]$

is bounded since so are all the signals on the righthand side of the previous equation. Thus, by Barbălat Lemma, $\lim _{t \rightarrow \infty} \dot{v}_{i}(t)=0$. This and (11), in turn, imply that $\lim _{t \rightarrow \infty} \ddot{\boldsymbol{\vartheta}}_{v i}(t)=0$ and that $\lim _{t \rightarrow \infty} \boldsymbol{\varphi}^{\top}\left(\theta_{i}(t)\right) \mathbf{e}_{i}(t)=0$.

Finally, we establish that $\varphi^{\perp \top}\left(\theta_{i}(t)\right) \mathbf{e}_{i}(t) \rightarrow 0$. To that end, we first compute the derivative of $\ddot{v}_{i}$; we have

$$
\begin{aligned}
v_{i}^{(3)}= & -\boldsymbol{\varphi}^{\top}\left(\theta_{i}\right)\left[d_{v i} \ddot{\boldsymbol{\vartheta}}_{v i}+p_{v i} \ddot{\mathbf{e}}_{i}\right] \\
& -2 \omega_{i} \boldsymbol{\varphi}^{\perp \top}\left(\theta_{i}\right)\left[d_{v i} \dot{\boldsymbol{\vartheta}}_{v i}+p_{v i} \dot{\mathbf{e}}_{i}\right] \\
& +\left[\omega_{i}^{2} \boldsymbol{\varphi}\left(\theta_{i}\right)-\dot{\omega}_{i} \boldsymbol{\varphi}^{\perp}\left(\theta_{i}\right)\right]^{\top}\left[d_{v i} \boldsymbol{\vartheta}_{v i}+p_{v i} \mathbf{e}_{i}\right] .
\end{aligned}
$$

We note that $v_{i}^{(3)}$ is bounded since so are all the terms on the right-hand side of the previous equation. Thus, $\ddot{v}_{i}$ is uniformly continuous and $\lim _{t \rightarrow \infty} \ddot{v}_{i}(t)=0$. Invoking systematically Barbălat Lemma one also obtains that $v_{i}^{(3)}, v_{i}^{(4)}$ and $\boldsymbol{\vartheta}_{v i}^{(3)}$ converge to zero.

Furthermore, from (13) it follows that the term $\omega_{i} \varphi^{\perp \top}\left(\theta_{i}\right) \mathbf{e}_{i}$ converges to zero. Moreover, on one hand, $\varphi^{\top}\left(\theta_{i}\right) \mathbf{e}_{i}=0 \Leftrightarrow \mathbf{e}_{i}=c \boldsymbol{\varphi}^{\perp}\left(\theta_{i}\right)$, for any $c \in \mathbb{R}$, and, on the other hand, $\varphi^{\perp \top}\left(\theta_{i}\right) \varphi^{\perp}\left(\theta_{i}\right)=1$, then it also follows that $\omega_{i}$ converges to zero. This in turn implies that $\vartheta_{\omega i}$ and that $\dot{\vartheta}_{\omega i}$ converge to zero; this holds for $\alpha_{i} \not \equiv 0$.

Finally, we compute

$$
\begin{aligned}
\ddot{\omega}_{i}=- & d_{\omega i} \dot{\vartheta}_{\omega i}-p_{\omega i} \dot{e}_{\theta i}-k_{\alpha i} \omega_{i} f_{i} \varphi^{\top}\left(\theta_{i}\right) \mathbf{e}_{i} \\
& +k_{\alpha i} f_{i} \boldsymbol{\varphi}^{\perp \top}\left(\theta_{i}\right) \dot{\mathbf{e}}_{i}+k_{\alpha i} \dot{f}_{i} \boldsymbol{\varphi}^{\perp \top}\left(\theta_{i}\right) \mathbf{e}_{i}
\end{aligned}
$$


and we replace it in

$$
\begin{aligned}
v_{i}^{(4)}= & -\boldsymbol{\varphi}^{\top}\left(\theta_{i}\right)\left[d_{v i} \boldsymbol{\vartheta}_{v i}^{(3)}+p_{v i} \mathbf{e}_{i}^{(3)}\right] \\
& -3 \omega_{i} \boldsymbol{\varphi}^{\perp \top}\left(\theta_{i}\right)\left[d_{v i} \ddot{\boldsymbol{\vartheta}}_{v i}+p_{v i} \ddot{\mathbf{e}}_{i}\right] \\
& +3\left[\omega_{i}^{2} \boldsymbol{\varphi}\left(\theta_{i}\right)-\dot{\omega}_{i} \boldsymbol{\varphi}^{\perp}\left(\theta_{i}\right)\right]^{\top}\left[d_{v i} \dot{\boldsymbol{\vartheta}}_{v i}+p_{v i} \dot{\mathbf{e}}_{i}\right] \\
& +\left[3 \omega_{i} \dot{\omega}_{i} \boldsymbol{\varphi}\left(\theta_{i}\right)+\omega_{i}^{3} \boldsymbol{\varphi}^{\perp}\left(\theta_{i}\right)\right]^{\top}\left[d_{v i} \boldsymbol{\vartheta}_{v i}+p_{v i} \mathbf{e}_{i}\right] \\
& -\ddot{\omega}_{i} \boldsymbol{\varphi}^{\perp \top}\left(\theta_{i}\right)\left[d_{v i} \boldsymbol{\vartheta}_{v i}+p_{v i} \mathbf{e}_{i}\right] .
\end{aligned}
$$

Note that, since $v_{i}, \omega_{i}, \vartheta_{\omega i}, \dot{\vartheta}_{\omega i}, \varphi^{\top}\left(\theta_{i}\right) \mathbf{e}_{i}$, and $v_{i}$ converge to zero all the terms on the right-hand side of (14), except for $\dot{f}_{i} \varphi^{\perp \top}\left(\theta_{i}\right) \mathbf{e}_{i}$, converge to zero. It follows that all terms on the right-hand side of (15) except for $p_{v i} k_{\alpha i} \dot{f}_{i}\left|\varphi^{\perp \top}\left(\theta_{i}\right) \mathbf{e}_{i}\right|^{2}$, tend to zero. Since, also, $v_{i}^{(4)} \rightarrow 0$ and $\lim _{t \rightarrow \infty} \dot{f}(t) \neq 0$, necessarily $\left|\varphi^{\perp \top}\left(\theta_{i}\right) \mathbf{e}_{i}\right|^{2} \rightarrow 0$. Thus, $\alpha_{i}$ vanishes and the limits in (4) follow.

\section{Simulations}

In order to illustrate our theoretical findings we performed some numerical simulations in Simulink ${ }^{\mathrm{TM}}$ of Matlab ${ }^{\mathrm{TM}}$. The simulation setup consists in six robots being required to meet at a rendez-vous point that is a priori unknown, but forming a pattern. In this case, an hexagon, that is defined by setting the offsets $\left(\delta_{x i}, \delta_{y i}\right)$ to the values showed in Table I; in the latter table, the initial conditions of each robot are also specified.

TABLE I

INITIAL CONDITIONS, RELATIVE DESIRED POSITIONS, AND DESIRED ORIENTATIONS.

\begin{tabular}{c|c|c|c|c|c|}
\cline { 2 - 6 } & $x_{i}(0)$ & $y_{i}(0)$ & $\theta_{i}(0)$ & $\delta_{x_{i}}$ & $\delta_{y_{i}}$ \\
\hline 1 & 2 & 5 & 0 & 2 & 0 \\
\hline 2 & 7 & 5.5 & $-\pi / 4$ & 1 & 2 \\
\hline 3 & 7 & 3.5 & $-p i / 2$ & -1 & 2 \\
\hline 4 & 3 & 2 & $\pi / 4$ & -2 & 0 \\
\hline 5 & 1 & 3.5 & $\pi / 2$ & -1 & -2 \\
\hline 6 & 3 & 2 & $\pi / 4$ & 1 & -2 \\
\hline
\end{tabular}

It is assumed that the graph topology is given by the Laplacian

$$
L:=\left[\begin{array}{rrrrrr}
3 & -1 & 0 & -1 & 0 & -1 \\
-1 & 2 & -1 & 0 & 0 & 0 \\
0 & -1 & 3 & -1 & 0 & -1 \\
-1 & 0 & -1 & 3 & -1 & 0 \\
0 & 0 & 0 & -1 & 2 & -1 \\
-1 & 0 & -1 & 0 & -1 & 3
\end{array}\right]
$$

and is illustrated in Fig. 3.

In Fig. 4 is appreciated the good performance of the closed-loop system; the robots converge to the desired hexagon-shaped formation. The final, coinciding, orientations of the robots are also depicted, represented by arrows. The time responses of the systems' trajectories are showed in Figs. 5 and 6. In the former, we show the time evolution

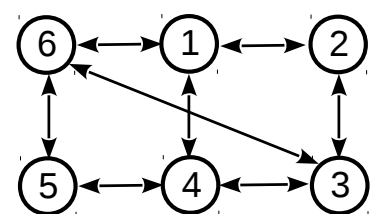

Fig. 3. Graph representation

of $\overline{\mathbf{z}}_{i}(t)$, which converges to the centre with coordinates $\left(x_{c}, y_{c}\right)=(-0.75,8.2)$ and in Fig. 6 we show the evolution of the angular orientations, which converge to the equilibrium $\theta_{c} \approx-1.35$.

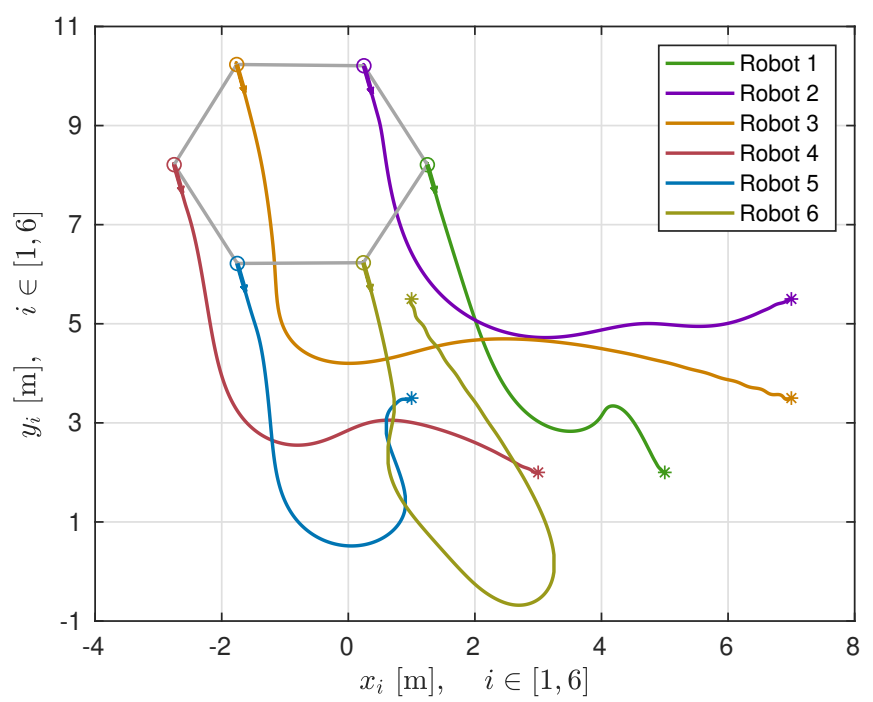

Fig. 4. Path followed by the robots on the plane
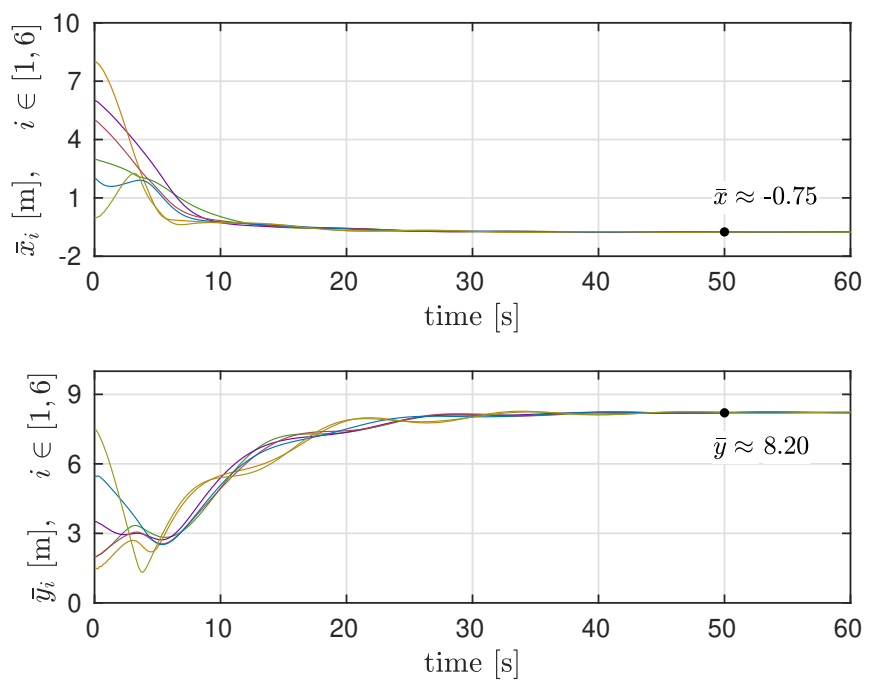

Fig. 5. Evolution of trajectories in Cartesian coordinates 




Fig. 6. Evolution of the orientation angles

\section{CONCLUSiOnS}

This paper reports a novel controller for the consensus formation control of nonholonomic robots that do not have velocity sensors. The stabilization technique relies, on one hand, on a dynamic controller that injects damping through an approximate-derivatives filter and back-propagates it throughout the plant. On the other hand, to deal with the nonholonomic restrictions, a $\delta$-persistently-exciting term is properly designed. Assuming that the interconnection graph is static, undirected and connected, the proposed scheme ensures consensus both in position and orientation for arbitrary initial conditions. Present research efforts are focused on the design of output-feedback consensus controllers in the presence of measurement delay.

\section{APPENDIX}

The proofs rely on the following expectable statement for which we present a short proof, for the sake of completeness.

Lemma 1: Consider system

$$
\begin{aligned}
& \dot{\mathbf{x}}=-a \mathbf{x}+\mathbf{u}, \\
& \mathbf{y}=\mathbf{x},
\end{aligned}
$$

where $a>0$ and $\mathbf{x}, \mathbf{u}, \mathbf{y} \in \mathbb{R}^{n}$ are the state, the input and the output, respectively. If $\mathbf{y} \in \mathcal{L}_{\infty} \cap \mathcal{L}_{2}$ and $\mathbf{u}, \dot{\mathbf{u}} \in \mathcal{L}_{\infty}$, then $\lim _{t \rightarrow \infty} \mathbf{u}(t)=\mathbf{0}$.

Proof: Clearly, from (16), one has that $\mathbf{y}, \mathbf{u} \in \mathcal{L}_{\infty}$ implies that $\dot{\mathrm{x}} \in \mathcal{L}_{\infty}$. This last and the fact $\mathrm{x} \in \mathcal{L}_{\infty} \cap \mathcal{L}_{2}$ ensures, by Barbălat Lemma, $\lim _{t \rightarrow \infty} \mathbf{x}(t)=\mathbf{0}$. Moreover,

$$
\lim _{t \rightarrow \infty} \int_{0}^{t} \dot{\mathbf{x}}(\sigma) d \sigma=\lim _{t \rightarrow \infty} \mathbf{x}(t)-\mathbf{x}(0)=-\mathbf{x}(0)
$$

and $\ddot{\mathrm{x}} \in \mathcal{L}_{\infty}$ because $\dot{\mathrm{x}}, \dot{\mathrm{u}} \in \mathcal{L}_{\infty}$. Therefore, invoking again Barbălat Lemma, [24, Lemma 8.2], we have that $\lim _{t \rightarrow \infty} \dot{\mathbf{x}}(t)=$ 0. Hence, $\lim _{t \rightarrow \infty} \mathbf{u}(t)=\mathbf{0}$, as required.

\section{REFERENCES}

[1] A. Jadbabaie, J. Lin, and A.S. Morse. Coordination of groups of mobile autonomous agents using nearest neighbor rules. IEEE Transactions on Automatic Control, 48(6):988-1001, 2003.

[2] R. Olfati-Saber and R.M. Murray. Consensus problems in networks of agents with switching topology and time-delays. IEEE Transactions on Automatic Control, 49(9):1520-1533, 2004.
[3] E. Nuño, R. Ortega, L. Basañez, and D. Hill. Synchronization of networks of nonidentical Euler-Lagrange systems with uncertain parameters and communication delays. IEEE Transactions on Automatic Control, 56(4):935-941, 2011.

[4] Y. Cao and W. Ren. Distributed Coordination of Multi-agent Networks: Emergent Problems, Models, and Issues. Springer-Verlag, 2011.

[5] H. Wang. Consensus of networked mechanical systems with communication delays: A unified framework. IEEE Transactions on Automatic Control, 59(6):1571-1576, 2014.

[6] T. Hatanaka, N. Chopra, M. Fujita, and M.W. Spong. Passivity-Based Control and Estimation in Networked Robotics. Communications and Control Engineering. Springer, 2015.

[7] Z. Lin, B. Francis, and M. Maggiore. Necessary and sufficient graphical conditions for formation control of unicycles. IEEE Transactions on Automatic Control, 50(1):121-127, 2005.

[8] D.V. Dimarogonas and K.J. Kyriakopoulos. On the rendezvous problem for multiple nonholonomic agents. IEEE Transactions on Automatic Control, 52(5):916-922, 2007.

[9] C. Yang, W. Xie, C. Lei, and B. Ma. Smooth time-varying formation control of multiple nonholonomic agents. In Proceedings of the 2015 Chinese Intelligent Systems Conference, pages 283-291. Springer, 2016.

[10] A. Ajorlou, M.M. Asadi, M.G. Aghdam, and S. Blouin. Distributed consensus control of unicycle agents in the presence of external disturbances. Systems \& Control Letters, 82:86-90, 2015.

[11] W. Dong and J.A. Farrell. Consensus of multiple nonholonomic systems. In Decision and Control, 2008. CDC 2008. 47th IEEE Conference on, pages 2270-2275. IEEE, 2008.

[12] Y. Cheng, R. Jia, H. Du, G. Wen, and W. Zhu. Robust finitetime consensus formation control for multiple nonholonomic wheeled mobile robots via output feedback. International Journal of Robust and Nonlinear Control, 28(6):2082-2096, 2018.

[13] E. Nuño, T. Hernández, M. Maghenem, A. Loría, and E. Panteley. Leaderless consensus-based formation control of multiple nonholonomic mobile robots with interconnecting delays. In Proc. IEEE American Control Conference, pages 4659-4664, Philadelphia, PA, USA, 2019.

[14] M. Maghenem, A. Bautista-Castillo, E. Nuño, A. Loría, and E. Panteley. Consensus of multi-agent systems with nonholonomic restrictions via Lyapunov's direct method. IEEE Control Systems Letters, 3(2):344-349, 2019.

[15] R. Kelly. A simple set-point robot controller by using only position measurements. In 12th. IFAC World Congress, volume 6, pages 173 176, Sydney, Australia, 1993.

[16] A. Loría. Observers are unnecessary for output-feedback control of Lagrangian systems. IEEE Trans. on Automatic Control, 61(4):905920, 2016.

[17] E. Nuño and R. Ortega. Achieving consensus of Euler-Lagrange agents with interconnecting delays and without velocity measurements via passivity-based control. IEEE Transactions on Control Systems Technology, 26(1):222-232, 2018.

[18] E. Panteley, A. Loría, and A. Teel. Relaxed persistency of excitation for uniform asymptotic stability. IEEE Trans. on Automat. Contr., 46(12):1874-1886, 2001.

[19] A. Loría, E. Panteley, and A. Teel. A new persistency-of-excitation condition for UGAS of NLTV systems: Application to stabilization of nonholonomic systems. In Proc. 5th. European Contr. Conf., pages 1363-1368, Karlsrühe, Germany, 1999.

[20] T. C. Lee, Kai. T. Song, C. H. Lee, and C. C. Teng. Tracking control of unicycle-modeled mobile robots using a saturation feedback controller. IEEE Transactions on Control Systems Technology, 9(2):305-318, Mar 2001.

[21] R. W. Brockett. Asymptotic stability and feedback stabilization. Differential geometric control theory, 27(1):181-191, 1983.

[22] A. Loría. From feedback to cascade-interconnected systems: Breaking the loop. In Proc. 47th. IEEE Conf. Decision Contr., pages 4109-4114, Cancun, Mex., 2008.

[23] H. Wang. Integral-cascade framework for consensus of networked Lagrangian systems. Technical report, 2018.

[24] H.K. Khalil. Nonlinear Systems. Prentice-Hall, New Jersey, third edition, 2002. 